\title{
Do More Powerful Interest Groups Have a Disproportionate Influence on Policy?
}

\author{
Zara Sharif ${ }^{1}$. Otto H. Swank ${ }^{2}$ (I)
}

Published online: 11 April 2019

(c) The Author(s) 2019

\begin{abstract}
Decisions-makers often rely on information supplied by interested parties. In practice, some parties have easier access to information than other parties. In this light, we examine whether more powerful parties have a disproportionate influence on decisions. We show that more powerful parties influence decisions with higher probability when their stakes are sufficiently large. However, in expected terms, decisions do not depend on the relative strength of interested parties. When parties have not provided information, decisions are biased towards the less powerful parties. Finally, we show that compelling parties to supply information destroys incentives to collect information.
\end{abstract}

Keywords Information collection - Communcation · Interest groups · Decisionmaking

JEL Classification D72 $\cdot$ D78 $\cdot$ D82 $\cdot$ H39

Practical politicians and journalists have long understood that small 'special interest' groups, the 'vested interests', have disproportionate power...[a group] will sometimes attain its objective even if the vast majority of the population loses as a result.

- Mancur Olson, The Logic of Collective Action, p. 127-128.

Otto H. Swank

swank@ese.eur.nl

1 Erasmus School of Economics and Tinbergen Institute, Erasmus University Rotterdam, E1-13, P.O. Box 1738, 3000 DR Rotterdam, The Netherlands

2 Erasmus School of Economics and Tinbergen Institute, Erasmus University Rotterdam, E1-32, P.O. Box 1738, 3000 DR Rotterdam, The Netherlands 
There will be no economic or social questions that would not be political questions in the sense that their solution will depend exclusively on who wields the coercive power, on whose are the views that will prevail on all occasions.

— Friedrich A. Hayek, The Road to Serfdom, p. 107.

So that the population of these civilized countries now falls into two main classes: those who own wealth invested in large holdings and who thereby control the conditions of life for the rest; and those who do not own wealth in sufficiently large holdings, and whose conditions are therefore controlled by these others...It is a division between the vested interests and the common man.

- Thorstein Veblen, The Vested Interests and The Common Man, p. 160-161.

All privileged and powerful classes, as such, have used their power in the interest of their own selfishness...

— John Stuart Mill, Principles of Political Economy, Book IV, p. 133.

\section{Introduction}

In June 2018, the Dutch government and energy companies Shell and ExxonMobil reached an agreement to gradually reduce and eventually stop gas extraction in the Northern Netherlands. This agreement was a response to hundreds of earthquakes associated with gas extraction. ${ }^{1}$ One year later, Dutch Parliament demanded a thorough investigation into past Dutch gas policies. The main question this investigation is supposed to address is how the Dutch government has weighed the interests of the citizens living close to the gas fields against the interests of the large energy companies. In the background of this question was the fight between two lobby groups with unequal power. Specifically, in 2013, after having learnt that gas extraction has caused hundreds of earthquakes, the Dutch government gave permission to the energy companies to extract the highest level of gas in 30 years. Only in 2018, an agreement has been reached more in line with the citizens' interests. However, for a long time the energy companies have been permitted to increase gas extraction in an area plagued with the adverse consequences of this extraction.

In a wide variety of situations, people make decisions on the basis of information supplied by other people. Often those who provide information have a "stake" in the final decision. One example is the one discussed above, where every year the Dutch government decides how much gas energy companies are allowed to extract in Groningen, and interested parties try to influence these decisions. Another prominent example of such a situation is a civil lawsuit involving a dispute between two parties about a distributional issue. Each party supplies information in an attempt

\footnotetext{
1 Several hundreds of earthquakes have been reported in Nothern Netherlands. Most of them are associated with gas extraction (Foulger et al. 2018). The largest was in 2012, an $M_{L} 3.6$ event. The first one was reported in 1991.
} 
to influence the judge's decision in its own favor. Another well-known example is a politician who makes a decision that affects various interest groups. Again each group may provide information with an eye on influencing the politician's final decision to its own benefit. When decisions are made on the basis of information provided by interested parties, there are usually two (related) concerns. First, interested parties have incentives to reveal information that is favorable for them, but to conceal information that is unfavorable for them. As a result, the decision maker possibly does not hear all available information. Second, the means of interest groups vary widely. An implication is that decisions may be biased towards the interests of groups with easier access to information.

The main objective of this paper is to shed light on these two concerns. To this end, we develop a game-theoretical model in which a neutral person has to resolve a distributional dispute between two parties; say, an amount of money is to be distributed. The socially optimal decision depends on the state of the world. The parties, however, have opposite interests that do not depend on the state of the world. As to learning the state, the decision maker has to rely on information provided by the parties. We assume that the parties do not observe the state of the world, ${ }^{2}$ but each party can exert effort to find verifiable information about it. The more effort a party puts in collecting information, the higher is the probability that a party receives verifiable information about the state. If information is found, a party has to determine whether to reveal or conceal it. An important feature of our model is that parties may differ in the (marginal) cost they attach to exerting effort. The implication is that there is a relatively advantaged party and a relatively disadvantaged party. In this way, we are able to address the concern regarding the influence of powerful interest groups on decisions. Another important feature of our model is that given the available information, the decision maker aims at making the socially optimal decision.

We derive four main results. The first one is neither novel nor surprising. Parties reveal information that promotes their interests, but conceal information that damages their interests.

Our second result is more subtle. The party that is relatively advantaged in terms of collecting information has stronger incentives to reveal it. The reason for this result is that when the advantaged party does not reveal information, the decision maker is inclined to believe that the party has something to hide. As a result, when neither party presents evidence, the decision is biased towards the interest of the disadvantaged party.

Third, in expected terms, the final decision does not depend on the relative strength of the parties. This neutrality result sheds light on the role of powerful interest groups in politics. Our model predicts that indeed relatively powerful interest groups frequently provide information that shapes policy. However, our model also predicts that if powerful interest groups do not provide information, decisions are made against their interests. In expected terms, these effects cancel out because of the Martingale property.

\footnotetext{
${ }^{2}$ In Sect. 8 we show that our main results also hold when parties observe the state of the world but must exert effort to communicate information.
} 
Our final result is that a policy that compels parties to reveal information destroys their incentives to collect information.

Together our results indicate that the concern that interested parties have incentives to conceal information is justified. However, compelling parties to supply information does not help. It would only weaken incentives to collect information. The concern for biased decisions because some parties have easier access to information than others is less justified. Rational decision makers take the relative strength of parties into account in such a way that differences in the power of parties do not lead to biases in decisions.

It is important to point out from the outset that we obtain our results from a model of informational lobbying in which the decision maker is unbiased. Of course, if the decision maker is biased or can be bribed our result that in expected terms the relative power of parties is irrelevant does not hold any more.

\section{Literature}

Our paper is related to two broad strands of economic literature. First is the literature on law and economics; researchers have investigated attorneys' incentives to collect and convey information in adversarial systems. An early paper is by Milgrom and Roberts (1986) who show that communication between interested parties with opposed interests leads to full-information decisions. Crucial assumptions for this result are (1) that information can be credibly transmitted, and (2) that parties are fully informed. When parties are not always fully informed, full revelation disappears (Austin-Smith 1994; Shin 1994). Dewatripont and Tirole (1999) show that parties with opposing preference have also strong incentives to collect information (Dur and Swank 2005; Kim 2014). In the literature on adversarial systems, our paper is closest to Sobel (1985), who examines parties' incentives to report information in case of a dispute over an indivisible asset. As in our paper, in Sobel one party might be more advantageous in reporting information than the other party. Sobel examines how different rules of proof of evidence affect parties' incentives. Our paper deviates from Sobel in that we focus on a dispute over a divisible asset. Moreover, we explicitly distinguish between incentives to collect information and incentives to transfer information.

Second, our paper is related to the voluminous literature on interest groups. ${ }^{3}$ Olson (1965) argues that smaller groups face lower costs to organize themselves, and consequently may have a disproportionate influence on policy. In Tullock (1980) and Becker (1983) interest groups decide how many resources to spend on lobbying. The amount of resources affects the probability of influencing the decision. It is this type of literature that predicts that an interest group with more resources has a bigger say in policy decisions. The early literature on lobbying posits the existence of an influence function describing how lobbying efforts affect policy. Potters and van Winden (1992) provide a micro-foundation for these influence functions. A

\footnotetext{
${ }^{3}$ For surveys, see Mitchell and Munger (1991), Mueller (2003) and Austen-Smith (1997).
} 
key assumption of their model is that an interest group possesses information that is relevant for a legislator. By paying a cost an interest group can credibly transmit information to the legislator. Potters and Van Winden show that the more the preferences of the interest group and the legislator are aligned, the wider is the scope for information transmission. ${ }^{4}$ Austen-Smith and Wright (1992), like us, model two groups that try to influence the decision of a legislator. Each group decides whether or not to become informed. This decision is observed by the legislator. Next, the two groups send messages to the legislator who makes the final decision. Our model deviates from Austen-Smith and Wright in three main respects. First, in our model, the decision and states are continuous rather than binary. Second, in our model, the decision-maker does not observe whether or not parties are informed. Finally, one of the main questions we address is whether more powerful interest groups have a bigger say in decisions, whereas the model by Austen-Smith and Wright is very suitable for understanding groups decisions on whether to lobby or not.

Similarly, some studies consider a group's choice of whether to use informational lobbying for influence or whether to use an alternative instrument such as campaign contributions (Bennedsen and Feldmann 2006) or political pressure (Dahm and Porteiro 2008). These models, though closely related to ours, are more suitable for understanding groups choice of the type of instrument to use for influence. Cotton (2012) considers the question of whether rich groups have a disproportionate influence on policy in a model where contributions determine access to the politician. They show that rich interest groups gain more access than poor groups, but that they are not better off compared to poor groups due to the politicians rent extracting strategy. While Cotton's focus is on the influence of strong groups due to better access to politicians, our focus is on the influence due to better information collection capabilities. Grossman and Helpman (2001) develop a cheap-talk model where interest groups are fully informed, but information is not verifiable. ${ }^{5}$ Their model too is more suitable to understand group decisions on whether to lobby or not. Moreover, their focus lies on the requirements for credibility when talk is cheap. They show that credibility improves with the amount of resources a group spends and thus provide a rationale for why interest groups spend more than is necessary to communicate messages. Lastly, common knowledge of the marginal cost of information collection allows the decision maker in our model to make an unbiased decision, which points towards the benefits of lobbying disclosure laws. In contrast, Denter et al. (2014) model lobbying as a contest between groups to show that mandated transparency of lobbying costs leads to an over-investment by groups and decreases expected allocative efficiency.

\footnotetext{
${ }^{4}$ See also Grossman and Helpman (2001).

5 See also Krishna and Morgan (2001) and Visser and Swank (2007).
} 


\section{The Model}

Our model describes a situation where a decision has to be made with important distributional consequences. One can think of, for example, the allocation of a tax. We assume that it is common knowledge that there is a socially optimal decision in the sense that reasons may exist why one party should be favored to the detriment of another party. To learn these reasons, the decision maker relies on the information supplied by the interested parties. We consider a setting in which each party wants to make a case for itself.

A decision maker has to choose $x$, where $x \in[l, h]$. One can think of the decision maker as a politician, a CEO, or a judge. The problem is that the proper decision is uncertain. This uncertainty is reflected by the stochastic term $\mu$, the state of the world, which is uniformly distributed ${ }^{6}$ on the interval $[l, h]$. The decision maker chooses $x$ so as to minimize the expected deviation of $x$ from $\mu$, given the information $(I)$ it possesses: $\min _{x}: E(|x-\mu| \mid I)$.

To learn $\mu$, the decision maker has to rely on information provided by two interested parties, $i \in\{a, b\}$. One can think of a party as an interest group, a manager of a division, or an attorney. Neither party knows $\mu$ initially. However, each party may collect information to learn $\mu$ and receive a signal $s_{i} \in\{\phi, \mu\}$. Collecting information is costly. Specifically, we assume that each party $i$ chooses effort $\pi_{i} \in[0,1)$, where $\pi_{i}$ denotes the probability with which party $i$ finds verifiable information about $\mu, s_{i}=\mu$. With probability $1-\pi_{i}$ party $i$ does not find information, $s_{i}=\phi$. For simplicity, we assume that the cost of information collection is quadratic: $\frac{1}{2} \lambda_{i} \pi_{i}^{2}$, with $\lambda_{i}>\frac{1}{2}(h-l)^{7}$

An important feature of our model is that $\lambda_{a}$ may differ from $\lambda_{b}$. If $\lambda_{a}<\lambda_{b}$, we say that party $a$ is the more powerful party. The parameter $\lambda_{i}$ may capture a few things. First, $\lambda_{i}$ may depend on the resources party $i$ possesses to collect information. Second, the efficiency with which a party collects information may affect $\lambda_{i}$. Third, $\lambda_{i}$ may depend on party $i$ 's position in the economy. For instance, information about the impact of a deregulation in an industry often lies in the hands of that industry. In this paper, we take a broad view of the various factors that may determine $\lambda_{i}$.

We assume that the two parties have opposing preferences. Party $a$ wants the decision maker to choose a high value of $x$, whereas party $b$ wants the decision maker to choose a low value of $x$. The payoffs to party $a$ and $b$ are given by:

$$
U_{a}(x)=x-\frac{1}{2} \lambda_{a} \pi_{a}^{2}
$$

and

\footnotetext{
6 The uniform distribution does not alter our results qualitatively. See also footnote 9.

7 In the "Appendix" we show that if $\lambda_{i} \leq \frac{1}{2}(h-l)$, an equilibrium exists in which party $i$ chooses $\pi_{i}=1$ and always reveals information to the decision maker. As a result, party $-i$ is redundant. By assuming $\lambda_{i}>\frac{1}{2}(h-l)$, we ensure that the model focuses on environments where both parties have incentives to collect information. This is the most relevant environment to investigate how the relative strength of parties affects decisions.
} 


$$
U_{b}(x)=-x-\frac{1}{2} \lambda_{b} \pi_{b}^{2}
$$

respectively.

After the parties have collected information, the communication stage starts. In this stage, the two parties simultaneously send a message, $m_{i}$, to the decision maker. A party conditions its message on the information it received, $m_{i}\left(s_{i}\right)$. We assume that information cannot be forged but can be concealed. Thus, if party $i$ did not find information in the collection stage, it cannot supply information, $m_{i}(\phi)=\phi$. If, by contrast, party $i$ found information, say $s_{i}=\mu^{\prime}$, it either sends $m_{i}\left(\mu^{\prime}\right)=\mu^{\prime}$ (reveals) or sends $m_{i}\left(\mu^{\prime}\right)=\phi$ (conceals). After the parties have sent their messages, the decision maker chooses $x$.

We assume that the structure of the game and the distribution of $\mu$ is common knowledge. Our model is a dynamic game with imperfect information. We solve it by backward induction and identify Perfect Bayesian Equilibria (PBE). The decision maker chooses $x$ so as to minimize $E\left(|x-\mu| \mid m_{a}, m_{b}\right)$. Parties anticipate the decision maker's decision rule.

\section{The Communication Stage}

Each party enters the communication stage either with the possibility to present evidence to the decision maker or without this possibility. This depends on whether or not a party was successful in the information collection stage. We call a party that is able to reveal information "informed", and a party that is not able "uninformed". By assumption, an uninformed party sends $m_{i}(\phi)=\phi$. The question remains for which values of $\mu$ an informed party sends $m_{i}(\mu)=\phi$ and for which values of $\mu$ it sends $m_{i}(\mu)=\mu$. Proposition 1 presents the equilibrium communication strategy of an informed party.

Proposition 1 In a PBE, parties' communication strategies can be characterized by a single threshold, $\mu^{T}$. An informed party a chooses $m_{a}(\mu)=\mu$ if and only if $\mu \geq \mu^{T}=E\left(\mu \mid m_{a}=m_{b}=\phi\right)$. An informed party $b$ chooses $m_{b}(\mu)=\mu$ if and only if $\mu \leq \mu^{T}$.

Proposition 1 is an implication of our assumption that the parties have opposing preferences. Information that is favorable for party $a$ is unfavorable for party $b$, and vice versa. At $\mu=\mu^{T}$, both parties are indifferent between revealing information $\left(m_{i}(\mu)=\mu\right)$ and concealing it $\left(m_{i}(\mu)=\phi\right)$. The decision of a party to reveal information or not is only relevant in case the other party does not reveal information. As the decision maker chooses $x=\mu$ if either party reveals information, $m_{i}\left(s_{i}\right)$ is not relevant if $m_{-i}(\mu)=\mu$. So, to determine party $a$ 's decision whether or not to report information, suppose $m_{b}\left(s_{b}\right)=\phi$ and $s_{a}=\mu^{\prime} \in\{l, h\}$. Clearly, $m_{a}\left(\mu^{\prime}\right)=\phi$ induces the decision maker to choose $x=E\left(\mu \mid m_{a}=m_{b}=\phi\right)$, while $m_{a}\left(\mu^{\prime}\right)=\mu^{\prime}$ induces the 
decision maker to choose $x=\mu^{\prime}$. Hence, party $a$ is indifferent between $m_{a}\left(\mu^{\prime}\right)=\mu^{\prime}$ and $m_{a}\left(\mu^{\prime}\right)=\phi$ if,

$$
\mu^{\prime T}=E\left(\mu \mid m_{a}=m_{b}=\phi\right)
$$

For party $b$, the same equation can be derived.

A direct implication of Proposition 1 is that in case both parties are able to provide evidence, the decision maker makes the full-information decision. This result is similar to the result derived by Milgrom and Roberts (1986) that competition between informed parties whose preferences are opposed leads to full-information decisions. Proposition 1 also implies that parties never provide evidence that conflicts with their own interests.

\section{Information Collection}

We now turn to a party's decision on how much effort to put in collecting verifiable information. Consider party $a$. When choosing $\pi_{a}$ party $a$ anticipates that it will only reveal information in the communication stage if $\mu \geq \mu^{T}$. Moreover, it anticipates that if party $b$ finds information, it will reveal it if and only if $\mu \leq \mu^{T}$. Finally, it knows that revealing $\mu$ leads to $x=\mu$. The expected payoff to party $a$ when choosing $\pi_{a}$ equals,

$$
\begin{aligned}
& \operatorname{Pr}\left(\mu \geq \mu^{T}\right)\left[\pi_{a} \frac{1}{2}\left(h+\mu^{T}\right)+\left(1-\pi_{a}\right) \mu^{T}\right]+\cdots \\
& \operatorname{Pr}\left(\mu \leq \mu^{T}\right)\left[\pi_{b} \frac{1}{2}\left(\mu^{T}+l\right)+\left(1-\pi_{b}\right) \mu^{T}\right]-\frac{1}{2} \lambda_{a} \pi_{a}^{2}
\end{aligned}
$$

The first (second) term of (4) pertains to the range of $\mu$ for which party $a(b)$ reveals information if it is found. The third term gives the cost of effort.

Differentiating (4) with respect to $\pi_{a}$, and using $\operatorname{Pr}\left(\mu \geq \mu^{T}\right)=\frac{h-\mu^{T}}{h-l}$ and $\operatorname{Pr}\left(\mu \leq \mu^{T}\right)=\frac{\left(\mu^{T}-l\right)}{h-l}$, we attain 8

$$
\pi_{a}=\frac{\left(h-\mu^{T}\right)^{2}}{2 \lambda_{a}(h-l)}
$$

Equation (5) shows that the higher is the deviation of $\mu^{T}$ from $h$, the more effort party $a$ puts in collecting information. Of course, the reason for this result is that the deviation of $\mu^{T}$ from $h$ is directly related to the probability that party $a$ will utilize its information. To put it somewhat differently, party $a$ has stronger incentives to collect information when it anticipates that the information is likely to be favorable to its cause. Obviously, it also has stronger incentives when the cost of collecting information is small.

In a similar way, one can derive the amount of effort party $b$ exerts:

$$
\pi_{b}=\frac{\left(\mu^{T}-l\right)^{2}}{2 \lambda_{b}(h-l)}
$$

\footnotetext{
${ }^{8}$ Due to our assumption $\lambda_{i}>\frac{1}{2}(h-l)$, we have $\pi_{a}<1$.
} 
Note that party $b$ 's effort strategy is the converse of party $a$ 's strategy. When party $b$ anticipates that it is likely to find information that is favorable to its cause, it has strong incentives to collect information.

\section{The Threshold $\mu^{T}$}

In Sect. 4, we have identified the communication strategies of the two parties. In these strategies, the threshold $\mu^{T}$ plays an important role. Party $a$ reveals information if and only if it has found that $\mu \geq \mu^{T}$, while the opposite holds for party $b$. In the previous section, we have examined the incentives of parties to collect information. Again the threshold $\mu^{T}$ turned out to be important. In the present section, we use parties' strategies to determine the threshold $\mu^{T}$.

In Sect. 4, we have shown that the threshold $\mu^{T}$ equals the expected value of $x$, conditional on $m_{a}=\phi$ and $m_{b}=\phi$. The decision maker knows that if both parties had found information, one of them would have revealed it. He can therefore infer from $m_{a}=\phi$ and $m_{b}=\phi$ that at most one party found information. As a consequence, parties not revealing information can be a result of three events. First, party $a$ found information, but decided not to reveal it. Then, $\mu<\mu^{T}$. Second, party $b$ found information, but decided not to reveal it, so that $\mu>\mu^{T}$. Third, neither party found information. As $\pi_{a}$ and $\pi_{b}$ are independent of $\mu$, in the third event the expected value of $\mu$ equals $\frac{1}{2}(l+h)$. Together these events imply the following expression for $\mu^{T}$,

$$
\mu^{T}=\frac{\pi_{a}\left(1-\pi_{b}\right)\left(\frac{\mu^{T}-l}{h-l}\right) \frac{l+\mu^{T}}{2}+\pi_{b}\left(1-\pi_{a}\right)\left(\frac{h-\mu^{T}}{h-l}\right) \frac{\mu^{T}+h}{2}+\left(1-\pi_{a}\right)\left(1-\pi_{b}\right) \frac{l+h}{2}}{\pi_{a}\left(1-\pi_{b}\right)\left(\frac{\mu^{T}-l}{h-l}\right)+\pi_{b}\left(1-\pi_{a}\right)\left(\frac{h-\mu^{T}}{h-l}\right)+\left(1-\pi_{a}\right)\left(1-\pi_{b}\right)}
$$

which can be rewritten as,

$$
\left(\mu^{T}\right)^{2}\left(\pi_{a}-\pi_{b}\right)+2 \mu^{T}\left[h\left(1-\pi_{a}\right)-l\left(1-\pi_{b}\right)\right]-h^{2}\left(1-\pi_{a}\right)+l^{2}\left(1-\pi_{b}\right)=0
$$

To better understand how $\mu^{T}$ depends on $\pi_{a}$ and $\pi_{b}$, first suppose that $\pi_{a}=\pi_{b}$. Then, (8) reduces to $\mu^{T}=\frac{1}{2}(l+h)$. This implies that in the absence of information, the decision maker chooses a neutral decision when parties exert the same amount of effort. Now suppose $\pi_{a}=\pi_{b}$. Straightforward, but tedious, algebra shows that $\mu^{T}$ is increasing in $\pi_{b}$ and decreasing in $\pi_{a}$. A direct implication is that for $\pi_{a}>\pi_{b}$, in the absence of information, a decision is made that is biased against party $a$. The intuition is straightforward. If $\pi_{a}>\pi_{b}$, the decision maker attributes a relatively high probability to the event that party $a$ possesses information. Consequently, in case neither party provides information in the communication stage, the decision maker is especially suspicious that party $a$ wants to hide information. Likewise for $\pi_{b}>\pi_{a}$ and $m_{a}=\phi$ and $m_{b}=\phi$, a decision is made that is biased against party $b$.

The effect of $\pi_{a}=\pi_{b}$ on the decision on $x$ influences parties' incentives to collect information. Recall that party $a$ 's effort equals $\pi_{a}=\frac{\left(h-\mu^{T}\right)^{2}}{2 \lambda_{a}(h-l)}$. Clearly, the lower is $\mu^{T}$, 
the higher is $\pi_{a}$. Again, this effect has a clear intuition. Party $a$ anticipates that in case the decision maker does not receive information about $\mu$, he will make a decision that is biased against its interest. This gives a stronger incentive for party $a$ to collect information.

Proposition 2 In equilibrium, $\mu^{T}$ is implicitly determined by (8). If $\lambda_{i}<\lambda_{-i}$ and $m_{a}=m_{b}=\phi$, a decision is made that is biased against party $i$.

Proof See "Appendix".

Proposition 2 sheds a new light on the claim that powerful interest groups are able to put a stamp on policy. Our model predicts that indeed powerful interest groups frequently provide evidence that heavily influences policy. In this sense, it is true that powerful interest groups have a disproportionate influence on policy. However, we have also shown that in case a powerful interest group does not provide information, the decision is biased against its interest.

The next proposition shows that the relative strength of interest groups does not affect the expected decision on $x$.

Proposition 3 In expected terms, the value of $\lambda_{i}$ relative to $\lambda_{-i}$ does not affect the decision on $x$.

\section{Proof See "Appendix".}

Proposition 3 is a direct implication of the Martingale property and we interpret it as a neutrality result. Of course, when one of the assumptions of our model is relaxed the neutrality result may break down. ${ }^{9}$ For example, we have assumed that the decision maker knows the relative strength of parties. If the decision maker were to have a wrong perception of $\lambda_{i}$, the neutrality result would no longer hold. Underestimation of the relative strength of a party induces the decision maker, in expected terms, to choose a policy that is favorable for that party. It is also important to emphasize that the neutrality result only holds for informative lobbying. Evidently, allowing for bribes may alter our results since they will directly influence the preferences of the decision maker.

\section{Forcing Parties to Reveal Their Information}

In the previous sections we have analyzed incentives of parties to collect and supply information. We have shown that a party only reveals information that benefits its cause. In the current section we examine the implications of a policy that

\footnotetext{
${ }^{9}$ It is important to note that relaxing the assumption that $\mu$ is uniformly distributed does not break down this result. In the proof provided in the "Appendix", we show that neutrality holds for a general distribution function.
} 
forces each party to reveal its information, whether that information is favorable for it or not. Such a policy in our model is akin to the assumption that information cannot be concealed. Consequently, the communication strategy of party $i$ becomes: $m_{i}\left(s_{i}\right)=\mu$ for $s_{i} \in[l, h]$, and $m_{i}(\phi)=\phi$. Note that in this setting the expected value of $\mu$ when the decision maker does not receive information equals $E\left(\mu \mid m_{a}=\phi, m_{b}=\phi\right)=\frac{1}{2}(l+h)$.

The resulting model revolves around information collection. When choosing the amount of effort to exert, parties anticipate that any information they find will be revealed, leading to $x=\mu$. Thus, the expected payoff to party $a$ when choosing $\pi_{a}$ is,

$$
\left(1-\pi_{a}\right)\left(1-\pi_{b}\right)\left(\frac{h+l}{2}\right)+\left[1-\left(1-\pi_{a}\right)\left(1-\pi_{b}\right)\right]\left(\frac{h+l}{2}\right)-\frac{1}{2} \lambda_{a} \pi_{a}^{2}
$$

The first term is the expected payoff in case neither party finds information. The second term is the expected payoff in case either of the two (or both) parties find information. The last term is the cost of effort. The first-order condition with respect to $\pi_{a}$ implies that the amount of effort party a exerts is $\pi_{a}=0$. Similarly, one can show that party $b$ has no incentive to collect information. Hence, compelling parties to reveal their information completely eliminates their incentives to become informed. This brings us to Proposition 4.

Proposition 4 A policy that compels parties to reveal their information eliminates their incentives to collect information.

Proposition 4 casts doubts on rules in legal systems that compel prosecutors to disclose exculpatory evidence to the defendant. One primary purpose of these rules is to ensure that all parties go to trial with as much knowledge as possible. Our result suggests that these rules may have an unintended consequence of discouraging parties to collect information in the first place.

\section{Costly Communication}

So far, we have focused on a situation where parties have to exert effort to find information. An alternative situation is that parties have information but have to make effort to convey it to the decision maker. ${ }^{10}$ To analyze the latter case, we assume that when choosing their strategies on effort, parties know $\mu$. In the new model, $\pi_{i}$ denotes the probability that party $i$ is able to provide verifiable evidence to the decision maker, and $\lambda_{i}$ can be interpreted as a measure of party $i$ 's accessibility to the decision maker. Specifically, in the alternative game we have that (1) nature chooses $\mu$ and reveals it to the parties, but not to the decision maker; (2) each party chooses

\footnotetext{
${ }^{10}$ Empirical research suggests that interest groups expend resources to convey their messages to policy makers. For a review of empirical models of interest group influence see Potters and Sloof (1996) and Stratmann (2005).
} 
effort on the basis of $\mu, \pi_{i}(\mu)$; (3) if party $i$ is able to reveal information, it reveals it or conceals it; (4) the decision maker chooses $x$.

The assumption about the observability of $\mu$ does not have consequences for the strategies followed in the communication stage. The communication strategies can again be characterized by a single threshold, $\mu^{T}$. Each party only reveals information when it perceives that it will lead to a more favorable decision.

Incentives to exert effort, however, are different in the present model. Because each party observes the state, effort is conditional on the state. The more favorable is the state to party $i$, the stronger are its incentives to exert effort. ${ }^{11}$ Moreover, if $\mu \leq \mu^{T}$, party $a$ does not exert effort, and if $\mu \geq \mu^{T}$ party $b$ does not exert effort. Thus, either party $a$ or party $b$ tries to convey information.

The assumption about the observability of $\mu$ does not affect our main result that in expected terms, the relative power of parties does not influence the decision on $x$. Of course, the reason is that also in the present model the Martingale property implies that the expected value of $x$ equals $\frac{1}{2(l+h)}$.

\section{Conclusion}

Do more powerful interest groups have a disproportionate influence on policy? We have shown in this paper that in an environment where interest groups try to influence decisions by concealing or revealing information, the answer to this question is in the negative. By often providing information, more powerful interest groups do frequently influence policies. However, when they abstain from providing information, decisions are biased against their interests. In expected terms, these effects cancel out.

We regard our neutrality result as a benchmark. Interest groups may systematically affect policies in case the assumptions underlying our model are violated. For instance, we have assumed that the decision maker forms expectations in a rational way. In practice, this means that the decision maker should distinguish between cases where more powerful interest groups do not provide information and cases where less powerful interest groups do not provide information. Moreover, our neutrality result requires that the decision maker correctly assess the abilities of interest groups to collect information. Finally, we have ignored the possibilities that interest groups bribe decision makers and that decision makers may already have ideological preferences over policies.

Open Access This article is distributed under the terms of the Creative Commons Attribution 4.0 International License (http://creativecommons.org/licenses/by/4.0/), which permits unrestricted use, distribution, and reproduction in any medium, provided you give appropriate credit to the original author(s) and the source, provide a link to the Creative Commons license, and indicate if changes were made.

${ }_{11}$ Specifically, $\pi_{a}(\mu)=\frac{\mu-\mu^{T}}{\lambda_{a}}$ for $\mu>\mu^{T}$ and $\pi_{a}(\mu)=0$ for $\mu \leq \mu^{T}$, and $\pi_{b}(\mu)=\frac{\mu^{T}-\mu}{\lambda_{b}}$ for $\mu<\mu^{T}$ and $\pi_{b}(\mu)=0$ for $\mu \geq \mu^{T}$. 


\section{Appendix}

As mentioned in Sect. 3, we assume $\lambda_{i}>\frac{1}{2}(h-l)$ to ensure that both parties have an incentive to acquire information. If $\lambda_{i} \leq \frac{h-4}{2}$, then $\pi_{i}=1$ and the decision maker relies entirely on party $i$. To see this, suppose $\lambda_{i}^{2} \leq \frac{1}{2}(h-l)$. Suppose that if $m_{a}=\phi, \mu^{T}=l$. Then, party $a$ chooses $\pi_{a}$ so as to maximize,

$$
\pi_{a} \frac{(h+l)}{2}+\left(1-\pi_{a}\right) l-\frac{1}{2} \lambda_{a}\left(\pi_{a}\right)^{2}
$$

yielding

$$
\frac{1}{2}(h-l)=\lambda_{a} \pi_{a}
$$

Then, $\pi_{a}=1$ for $\lambda_{a} \leq \frac{1}{2}(h-l)$.

\section{Proof of Proposition 2}

First we show $\mu^{T}$ is decreasing in $\pi_{a}$ and increasing in $\pi_{b}$. (8) solves for,

$$
\mu^{T}=\left\{\begin{array}{ll}
\frac{1}{\pi_{b}-\pi_{a}}\left(h\left(1-\pi_{a}-\right)-l\left(1-\pi_{b}\right)-(h-l) \sqrt{\left(1-\pi_{a}\right)\left(1-\pi_{b}\right)}\right) & \text { if } \pi_{a} \neq \pi_{b} \\
\frac{1}{2}(h+l) & \text { if } \pi_{a}=\pi_{b}
\end{array}\right\}
$$

This implies,

$$
\frac{\partial \mu^{T}}{\partial \pi_{a}}=\frac{\sqrt{\left(1-\pi_{a}\right)\left(1-\pi_{b}\right)}}{\left(\pi_{b}-\pi_{a}\right)^{2}\left(1-\pi_{a}\right)}\left(\pi_{a}+\pi_{b}+2 \sqrt{\left(1-\pi_{a}\right)\left(1-\pi_{b}\right)}-2\right)
$$

The first term is positive. We need to show $\pi_{a}+\pi_{b}+2 \sqrt{\left(1-\pi_{a}\right)\left(1-\pi_{b}\right)}-2<0$ :

$$
\begin{aligned}
\pi_{a}+\pi_{b}+2 \sqrt{\left(1-\pi_{a}\right)\left(1-\pi_{b}\right)}-2 & <0 \\
4\left(\pi_{a}-1\right)\left(\pi_{b}-1\right) & <\left(2-\pi_{a}-\pi_{b}\right)^{2} \\
4\left(\pi_{a}-1\right)\left(\pi_{b}-1\right)-\left(2-\pi_{a}-\pi_{b}\right)^{2} & <0 \\
-\left(\pi_{a}-\pi_{b}\right)^{2} & <0
\end{aligned}
$$


Thus, $\frac{\partial\left(\mu^{T}\right)}{\partial \pi_{a}}<0$. Symmetry implies, $\frac{\partial\left(\mu^{T}\right)}{\partial \pi_{b}}>0$.

Next, we can show that $\pi_{a}>\pi_{b} \Leftrightarrow \mu^{T}<\frac{1}{2}(l+h)$ :

$\Longrightarrow$ : Assume $\pi_{a}>\pi_{b}$. Let $\mu^{T}=\frac{1}{2}(l+h)+e$, so $e<0$ implies $\mu^{T}<\frac{1}{2}(l+h)$.

Substituting in (10) gives us,

$$
\begin{gathered}
\frac{1}{\pi_{b}-\pi_{a}}\left(h\left(1-\pi_{a}\right)-l\left(1-\pi_{b}\right)-(h-l) \sqrt{\left(1-\pi_{a}\right)\left(1-\pi_{b}\right)}\right)=\frac{l+h}{2}+e \\
\frac{1}{\pi_{a}-\pi_{b}}\left(\pi_{a}+\pi_{b}+2 \sqrt{\left(\pi_{a}-1\right)\left(\pi_{b}-1\right)}-2\right)=e
\end{gathered}
$$

The first term on the left is positive and the second is negative. Thus, if $\pi_{a}>\pi_{b}$, then $e<0$ which implies $\mu^{T}<\frac{1}{2}(l+h)$.

$\Leftarrow$ : Assume $\mu^{T}<\frac{1}{2}(l+h)$. Then (10) reduces to,

$$
\begin{gathered}
\frac{1}{\pi_{b}-\pi_{a}}\left(h\left(1-\pi_{a}\right)-l\left(1-\pi_{b}\right)-(h-l) \sqrt{\left(1-\pi_{a}\right)\left(1-\pi_{b}\right)}\right)<\frac{1}{2}(l+h) \\
\frac{1}{\pi_{a}-\pi_{b}}\left(\pi_{a}+\pi_{b}+2 \sqrt{\left(\pi_{a}-1\right)\left(\pi_{b}-1\right)}-2\right)<0
\end{gathered}
$$

Since the second term on the left-hand side is negative, we must have $\pi_{a}>\pi_{b}$.

Lastly, we can show, $\lambda_{a}<\lambda_{b} \Leftrightarrow \mu^{T}<\frac{1}{2}(l+h)$.

$\Leftarrow$ : Assume $\mu^{T}<\frac{1}{2}(l+h)$. This implies $\pi_{a}-\pi_{b}>0$, thus,

$$
\begin{gathered}
\frac{\left(h-\mu^{T}\right)^{2}}{2 \lambda_{a}(h-l)}-\frac{\left(\mu^{T}-l\right)^{2}}{2 \lambda_{b}(h-l)}>0 \\
\lambda_{b}\left(h-\mu^{T}\right)^{2}-\lambda_{a}\left(\mu^{T}-l\right)^{2}>0 \\
\lambda_{b}\left(h-\frac{l+h}{2}-e\right)^{2}-\lambda_{a}\left(\frac{l+h}{2}+e-l\right)^{2}>0 \\
\left(\lambda_{b}-\lambda_{a}\right)\left(\frac{h-l+2 e}{2}\right)^{2}>0 \Longrightarrow \lambda_{b}>\lambda_{a}
\end{gathered}
$$

$\Longrightarrow$ : Assume $\lambda_{a}<\lambda_{b}$. Similar to the last derivation, we obtain,

$$
\pi_{a}-\pi_{b}=\frac{1}{2(h-l) \lambda_{a} \lambda_{b}}(e)^{2}\left(\lambda_{b}-\lambda_{a}\right)>0
$$

Thus, if $\lambda_{a}<\lambda_{b}$, then $\pi_{a}>\pi_{b}$, which implies $\mu^{T}<\frac{1}{2}(l+h)$. 


\section{Proof of Proposition 3}

We need to show $E(x)=E(\mu)$. For simplicity, assume $h=-l$. This does not alter our results. In fact, below we show that this result holds for any general distribution function. If $h=-l$, then Eq. (7) implies that $\mu^{T}$ is implicitly determined by,

$$
\left(\mu^{T}\right)^{2}\left(\pi_{a}-\pi_{b}\right)+2 \mu^{T} h\left(2-\pi_{a}-\pi_{b}\right)+h^{2}\left(\pi_{a}-\pi_{b}\right)=0
$$

We need to show $E(x)=E(\mu)=\frac{h+l}{2}=0$.

$$
\begin{aligned}
E(x)= & \pi_{a} \pi_{b} E(\mu)+\pi_{a}\left(1-\pi_{b}\right)\left(E\left(\mu \mid \mu>\mu^{T}\right)+\frac{\mu^{T}-l}{h-l} \mu^{T}\right)+\cdots \\
& \pi_{b}\left(1-\pi_{a}\right)\left(E\left(\mu \mid \mu<\mu^{T}\right)+\frac{h-\mu^{T}}{h-l} \mu^{T}\right)+\left(1-\pi_{a}\right)\left(1-\pi_{b}\right) \mu^{T}
\end{aligned}
$$

Substituting out $E(\mu)=0, E\left(\mu \mid \mu>\mu^{T}\right)=\frac{h-\mu^{T}}{h-l} \frac{\mu^{T}+h}{2}$, and $E\left(\mu \mid \mu<\mu^{T}\right)=\frac{\mu^{T}-l}{h-l} \frac{\mu^{T}+l}{2}$, and reducing the expression gives us,

$$
E(x)=\frac{1}{4 h}\left[\left(\mu^{T}\right)^{2}\left(\pi_{a}-\pi_{b}\right)+2 \mu^{T} h\left(2-\pi_{a}-\pi_{b}\right)+h^{2}\left(\pi_{a}-\pi_{b}\right)\right]
$$

Using (11) implies that $E(x)=0$.

General distribution Here we show that this result also holds for any distribution function. Assume that the random variable $\mu$ has a probability density function $f(\mu)$. Let $p=\operatorname{Pr}\left(\mu>\mu^{T}\right)=\int_{\mu^{T}}^{h} f(\mu) d \mu$. For this general case, Eq. (8) is given by,

$$
\mu^{T}=\frac{\pi_{a} \pi_{b}(1-p) E\left(\mu \mid \mu<\mu^{T}\right)+\pi_{b}\left(1-\pi_{a}\right) p E\left(x \mid \mu>\mu^{T}\right)+\left(1-\pi_{a}\right)\left(1-\pi_{b}\right) E(\mu)}{\pi_{a} \pi_{b}(1-p)+\pi_{b}\left(1-\pi_{a}\right) p+\left(1-\pi_{a}\right)\left(1-\pi_{b}\right)}
$$

which implies that the threshold is implicitly determined by the following,

$$
\begin{aligned}
{\left[1-p \pi_{a}-(1-p) \pi_{b}\right] \mu^{T}=} & \cdots \pi_{a}\left(1-\pi_{b}\right)(1-p) E\left(\mu \mid \mu<\mu^{T}\right) \\
& +\pi_{b}\left(1-\pi_{a}\right) p E\left(\mu \mid \mu>\mu^{T}\right) \\
& +\left(1-\pi_{a}\right)\left(1-\pi_{b}\right) E(\mu)
\end{aligned}
$$

Similarly, for the general case, the final decision in expectation is given by,

$$
\begin{aligned}
E(x)= & \pi_{a} \pi_{b} E(\mu)+\pi_{a}\left(1-\pi_{b}\right)\left[p E\left(\mu \mid \mu>\mu^{T}\right)+(1-p) \mu^{T}\right]+\cdots \\
& E \pi_{b}\left(1-\pi_{a}\right)\left[(1-p) E\left(\mu \mid \mu<\mu^{T}\right)+(1-p) \mu^{T}\right]+\left(1-\pi_{a}\right)\left(1-\pi_{b}\right) \mu^{T}
\end{aligned}
$$

which can be rewritten as,

$$
\begin{aligned}
E(x)= & \pi_{a} \pi_{b} E(\mu)+\pi_{a}\left(1-\pi_{b}\right) p E\left(\mu \mid \mu>\mu^{T}\right) \\
& +\pi_{b}\left(1-\pi_{a}\right)(1-p) E\left(\mu \mid \mu<\mu^{T}\right) \cdots \\
& +\left[1-p \pi_{a}-(1-p) \pi_{b}\right] \mu^{T}
\end{aligned}
$$


The last term in this expression can be substituted out using Eq. (13). Equation (15) reduces to,

$$
\begin{aligned}
E(x)= & E(\mu)+\left(\pi_{a}+\pi_{b}-2 \pi_{a} \pi_{b}\right) \\
& {\left[p E\left(\mu \mid \mu>\mu^{T}\right)+(1-p) E\left(\mu \mid \mu<\mu^{T}\right)-E(\mu)\right] }
\end{aligned}
$$

Note that $E(x)=E(\mu)$ if the second term on the right hand side equals zero. This follows directly from the law of total expectation, which states that we must have, $p E\left(\mu \mid \mu>\mu^{T}\right)+(1-p) E\left(\mu \mid \mu<\mu^{T}\right)=E(\mu)$, and thus, $E(x)=E(\mu)$.

\section{Proof Proposition 4}

See main text.

\section{References}

Austin-Smith, D. (1994). Strategic transmission of costly information. Econometrica, 62, 955-963.

Austen-Smith, D. (1997). Interest groups: Money, information and influence. In D. C. Mueller (Ed.), Perspectives on Public Choice. Cambridge: Cambridge University Press.

Austen-Smith, D., \& Wright, J. R. (1992). Competitive lobbying for a legislator's vote. Social Choice and Welfare, 9, 229-257.

Bennedsen, M., \& Feldmann, S. E. (2006). Informational lobbying and political contributions. Journal of Public Economics, 90(4), 631-656.

Becker, G. S. (1983). A theory of competition among pressure groups for political influence. The Quarterly Journal of Economics, 98, 371-400.

Cotton, C. (2012). Pay-to-play politics: Informational lobbying and contribution limits when money buys access. Journal of Public Economics, 96(3/4), 369-386.

Dahm, M., \& Porteiro, N. (2008). Informational lobbying under the shadow of political pressure. Social Choice and Welfare, 30, 531-559.

Denter, P., Morgan, J, \& Sisak D. (2014). Where ignorance is bliss, 'tis folly to be wise': Transparency in contests. In Working paper.

Dewatripont, M., \& Tirole, J. (1999). Advocates. Journal of Political Economy, 107, 1-39.

Dur, R. A. J., \& Swank, O. H. (2005). Producing and manipulating information. The Economic Journal, $115,185-199$

Foulger, G. R., Wilson, M. P., Gluyas, J. G., Julian, B. R., \& Davies, R. J. (2018). Global review of human-induced earthquakes. Earth-Science Reviews, 178, 438-514.

Grossman, G. M., \& Helpman, E. (2001). Special interest politics. Cambridge, MA: The MIT Press.

Kim, C. (2014). Adversarial and inquisitorial procedures with information acquisition. The Journal of Law, Economics, and Organization, 30(4), 767-803.

Krishna, V., \& Morgan, J. (2001). A model of expertise. The Quarterly Journal of Economics, 116, $747-775$.

Milgrom, P., \& Roberts, J. (1986). Relying on information of interested parties. The RAND Journal of Economics, 17, 18-32.

Mitchell, W. C., \& Munger, M. C. (1991). Economic models of interest groups: An introductory survey. Americal Journal of Political Science, 35, 512-546.

Mueller, Dennis C. (2003). Public choice III. Cambridge: Cambridge Books, Cambridge University Press.

Olson, M, Jr. (1965). The logic of collective action: Public goods and the theory of groups. Cambridge, MA: Harvard University Press.

Potters, J., \& van Winden, F. (1992). Lobbying and asymmetric information. Public Choice, 74, 269-292. 
Potters, J., \& Sloof, R. (1996). Interest groups: A survey of empirical models that try to assess their influence. European Journal of Political Economy, 12, 403-442.

Shin, H. S. (1994). The burden of proof in a game of persuasion. Journal of Economic Theory, 25, 253-263.

Sobel, J. (1985). Disclosure of evidence and resolution of disputes: Who should bear the burden of proof? In A. E. Roth (Ed.), Game theoretic models of bargaining, Ch. 16. Cambridge: Cambridge University Press.

Stratmann, T. (2005). Some talk: Money in politics. A (partial) review of the literature. Public Choice, $124,135-156$.

Tullock, G. (1980). Efficient rent-seeking. In J. M. Buchanan, R. D. Tollison, \& G. Tullock (Eds.), Towards a theory of rent-seeking society. College Station, TX: A\&M University Press.

Visser, B., \& Swank, O. H. (2007). On committees of experts. The Quarterly Journal of Economics, 122(1), 337-372.

Publisher's Note Springer Nature remains neutral with regard to jurisdictional claims in published maps and institutional affiliations. 